\title{
A Survey on Comparison of Various Protocols and Key Management Issues in Manet
}

\author{
S. Naga Mallik Raj ${ }^{1}$, S. Neeraja ${ }^{2}$, N. Thirupathi Rao ${ }^{3}$, Debnath Bhattacharyya ${ }^{4}$ \\ 1,3,4 Department of Computer Science and Engineering, Vignan's Institute of \\ Information Technology, Visakhapatnam, AP, India \\ ${ }^{2}$ Department of Computer Science and Engineering, Pydah Engineering College, \\ Visakhapatnam, AP, India \\ ${ }^{1}$ mallikblue@gmail.com, ${ }^{2}$ neerajasreerama@gmail.com, \\ ${ }^{3}$ nakkathiru@gmail.com ${ }^{4}$ debnathb@gmail.com
}

\begin{abstract}
Remote Network incorporates a bigger favorable position in the present correspondence application like ecological, movement, military and wellbeing perception. To understand these applications it's important to have a solid directing convention. The self-sorting out nature of MANETs makes them appropriate for some applications and henceforth, extensive exertion has been put into anchoring this kind of systems. Secure correspondence in a system is dictated by the unwavering quality of the key administration conspire, which is in charge of creating, circulating and looking after encryption/decoding keys among the hubs. In this paper different key administration plans for MANETs are talked about. This examination work proposes a novel secure Identity-Based Key Management convention making utilization of cryptographic and Information Theoretic Security.
\end{abstract}

Keywords: MANET, steering conventions, key administration, symmetric key, hilter kilter key, amass key administration.

\section{Introduction}

The ongoing advancement of specially appointed remote advances has permitted versatile impromptu systems (MANETs) to build unconstrained associations among cell phones with none foundation. Besides, with the development of sensor-empowered keen cell phones, MANETs turned into a fundamental part inside the foundation of shrewd city and web of Things (IoT) circumstances because of people with savvy gadgets will openly and powerfully kind a self-arranging MANET to send, get and share information in an exceedingly confined zone.

In an exceedingly such a keen situation, MANETs, WSNs and WMNs speak to key innovations giving numerous IoT applications and administrations to clients. Besides MANETs have discovered a scope of uses in human services, front line correspondences, debacle recuperation, emergency administration administrations instruction associations, impromptu agreeable registering, social exercises and gathering lobbies [1].

Article history:

Received (March 30, 2019), Review Result (May 11, 2019), Accepted (June 20, 2019) 
A MANET is an accumulation of self-governing hubs or terminals that speak with one another by shaping a multi-bounce radio system and keeping up network in a decentralized way. Because of the constrained transmission scope of every portable hub, it might be essential for one versatile hub to enroll the guide of different hubs in sending a bundle to its goal. Accordingly, in such condition, each hub in the system assumes the part of a switch by having the capacity to decide the ways of transmitting parcels to their goals. Figure 1 outlines a case of a MANET which contains two PCs, two PDAs and two computerized cameras. Since hub D is outside hub A's transmission extend, the information and retransmitted by hubs.

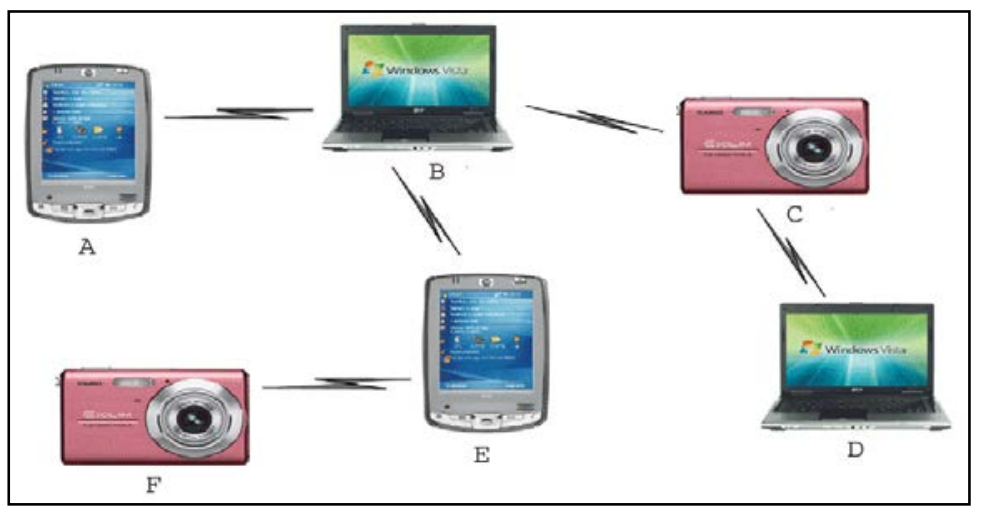

Figure 1. Mobile Adhoc Network

In spite of the appealing utilizations of MANETs, these frameworks still face a few difficulties and imperatives that need more examination before the across the board business arrangement of MANETs. The most requirements that may affect Manet configuration are as per the following: (1) the restricted vitality and lifetime of the battery, nature of administration (QoS),infrastructure-less and self-sufficient setup, dynamic system topologies, the versatility of hubs, remote connection unwavering quality, variety in hub abilities, multi-jump directing adaptability, multicast support and security dangers .

As it is hard to set up an ensured correspondence by key creation among flexible centers in a MANET, a malicious compact center point can use a phony character to make faked trust relations with various center points, and a short time later strike the MANET. Such center points would drop all of the data groups understood that they need to advance in the midst of whole reenactment. A solid steering convention for Mobile Ad hoc Networks (MANETs) keeps the vitality utilization as low as conceivable. Despite what might be expected, in MANET with cell organize joining, it is practical to validate portable hubs previously any real key age. Keeping in mind the end goal to build up trust connection between any two portable hubs in the cell based MANET, it is advantageous to exploit cell framework in order to empower a trustable and secure key age before correspondence.

\section{DIRECTING PROTOCOLS}

Directing is the demonstration of moving data from a source to a goal in an internetwork. Amid this procedure, not less than one middle of the road hub inside the internetwork is experienced.

The directing idea fundamentally includes, two exercises: right off the bat, deciding ideal steering ways and besides, exchanging the data gatherings (called bundles) through a web work. 
The later idea is called as bundle exchanging which is straight forward, and the way assurance could be exceptionally perplexing.

Directing conventions are grouped into various classifications relying upon their properties.

- Centralized versus Dispersed

- Static versus Versatile

- Reactive versus proactive

In incorporated estimations, all course choices are made at central center point, while in passed on computations, the count of the courses is shared among the framework center points another gathering of coordinating traditions relates to whether they change courses in light of the action input plans. In static computations, the course used by the source objective sets is settled paying little personality to development conditions. It can simply change in light of a center or association frustration. This kind of count can't achieve high throughput under a wide assortment of movement input designs.

Most significant bundle frameworks uses some sort of adaptable controlling where the courses used to course between source-objective sets may change in light of blockage . Proactive traditions reliably survey the courses inside the framework, with the objective that when a pack ought to be sent the course is starting at now known and can be in a flash used. Responsive traditions summon a course assurance methodology on request as it were. There is require another steering convention for correspondence arrange which incorporate versatile, adaptable, and secure viewpoints in it. A portion of the group based directing conventions are dissected beneath in Table I.

\section{SECURITY GOALS IN MANET}

When managing security in correspondence organize, one is looked with the issue of accomplishing a few or the greater part of the accompanying objectives:

- Availability: This implies organize resources are accessible to approved gatherings when required and system ought to guarantee the survivability of system administrations regardless of dissent of-benefit (DOS) assaults, which could be propelled at any layer of correspondence arrange.

- Genuineness: In correspondence arrange confirmation is fundamental for some authoritative errands (e.g. organize reinventing or controlling sensor hub obligation cycle). Information confirmation enables the collector to check that the information was extremely sent by the asserted sender. More grounded levels of validness (e.g. express key validation) are given by some key foundation conventions

- Confidentiality: A classified message is impervious to uncovering its importance to a meddler. Notwithstanding directing data in WAN needs to stay private, since it might be utilized to in a DOS assault. The standard answer for keep touchy information mystery is to encode the information with a mystery key that exclusive the expected recipients have, thus accomplishing privacy.

- Data uprightness: Integrity measures guarantee that they got information isn't modified in travel by an enemy. The uprightness administration can be furnished utilizing cryptographic hash works alongside some type of encryption. When managing system security, the honesty benefit is frequently given certainly by the validation benefit.

- Scalability and self-association: as opposed to general systems that don't place versatility in the principal need, WAN can't use a keying plan that has poor scaling properties (either regarding vitality cost or dormancy) for building up and keeping up a key for the WAN all in 
all or for some vast subset of hubs . As an outcome, the WAN hubs must have the capacity to self-compose and select the proper keying instrument for the circumstance.

Table 1. Investigation of Major Routing Protocols

\begin{tabular}{|c|c|c|}
\hline PROTOCOL & STRENGTH & DRAWBACKS \\
\hline LEACH & $\begin{array}{c}\text { It Improves the lifespan of networks, } \\
\text { Lowers the Energy consumption and it } \\
\text { takes the responsibility of cluster } \\
\text { heads. }\end{array}$ & $\begin{array}{l}\text { Selection of clusters } \\
\text { numbers were static } \\
\text { and Random }\end{array}$ \\
\hline $\begin{array}{l}\text { HYBRID ENERGY } \\
\text { EFFICIENT } \\
\text { DISTRIBUTED } \\
\text { PROTOCOL (HEED) }\end{array}$ & $\begin{array}{l}\text { HEED distribution of energy extends } \\
\text { the lifetime of the nodes within the } \\
\text { network thus stabilizing the } \\
\text { neighbouring node. } \\
\text { Does not require special node } \\
\text { capabilities, such as location-awareness }\end{array}$ & $\begin{array}{l}\text { The random } \\
\text { selection of the } \\
\text { cluster heads, may } \\
\text { cause higher } \\
\text { communication } \\
\text { overhead. }\end{array}$ \\
\hline $\begin{array}{c}\text { HIERARCHICAL } \\
\text { ENERGY EFFICIENT } \\
\text { ROUTING } \\
\text { PROTOCOL (HEERP) }\end{array}$ & $\begin{array}{c}\text { In dense networks also it performs } \\
\text { well. }\end{array}$ & $\begin{array}{c}\text { In less dense } \\
\text { network its } \\
\text { efficiency is very } \\
\text { low }\end{array}$ \\
\hline $\begin{array}{c}\text { HIERARCHICAL } \\
\text { GEOGRAPHIC } \\
\text { CLUSTERING } \\
\text { PROTOCOL (HGCP) } \\
\end{array}$ & It uses Virtual Grids & $\begin{array}{l}\text { With this protocol it } \\
\text { is assumed both the } \\
\text { sensors and actors } \\
\text { are static. }\end{array}$ \\
\hline LEACH - B & $\begin{array}{l}\text { It improves energy utilization, It } \\
\text { improves networks life cycle }\end{array}$ & Data fusion issue \\
\hline
\end{tabular}

Remote correspondence systems are testing a result of the unusual conduct of the medium and the proactive impact of impedance. Contrasted with the wired systems the level of inconstancy of the condition of remote systems is very high. Additionally the execution of the system, as far as postponement and throughput, is profoundly reliant upon the condition of the system. it is essential that the layers facilitate and adjust to the adjustment in arrange state. To manage the dynamic varieties in systems administration and processing assets nimbly, both the versatile registering condition and the applications likewise need to adjust their conduct contingent upon the accessible assets.

Late advances in the compactness, power, and abilities of remote gadgets and applications have brought about the expansion and expanded prominence of these gadgets. As the quantity of clients keeps on developing, remote steering conventions will be required to scale to progressively bigger populaces of hubs. Systems administration situations can require the arrangement of systems on the request of tens to several hubs, while numerous military applications can include thousands to a huge number of hubs. Moreover, as the arrangement of remote correspondence systems turns out to be more across the board, new applications may support the development of expansive correspondence systems. The primary target is to outline a safe steering convention for an extensive remote sensor arrange in which the hubs and in addition the base station are versatile. The convention ought to be secure, vitality proficient and versatile as for every single existing calculation.

\section{ENTER MANAGEMENT IN MANET}


Cryptography decreases the secrecy and respectability of a message to the privacy and uprightness of a key. When utilizing symmetric cryptography, the gatherings included need to arrange a mystery key. A decent key foundation conspire gives element validation (all gatherings know the personality of alternate gatherings with whom they are setting up a key), key verification (all gatherings are guaranteed that no unapproved gatherings could have gotten the mystery key), and key affirmation (all gatherings are guaranteed that every single other gathering know about the mystery key[7]

Key foundation plans can be separated into three noteworthy classifications:

(1) key pre-conveyance plans,

(2) plans utilizing a confided in outsider, and

(3) plans in view of open key cryptography.

Key pre-dispersion plans have gotten a considerable measure of consideration in the setting of advertisement ho c systems. They are extremely suited for advertisement ho c organizes as they don't require a confided in outsider to b e accessible consistently, and are exceptionally effective. Plans utilizing confided in outsiders are not so much suited for promotion ho c organizes as they accept that the confided in outsider is accessible to anyone. The detriment of open key based plans is that they require authentications and those open key calculations are in-effective.

Symmetric key calculations are computationally exceptionally productive and are along these lines of high enthusiasm for MANETs.

The system is apportioned into bunches. The hub with most extreme trust capacity is chosen as $\mathrm{CH}$. Among bunch individuals in a bunch, $\mathrm{k}$ hubs with high trust esteem are chosen as PKG serving hubs. Hubs can move starting with one bunch then onto the next. System manager chooses $\mathrm{CH}$. The disconnected CA appoints node_id for the hub that join the system. Each new hub has a self doled out open key and register its data in $\mathrm{CH}$. The private key of the hub is created by PKG serving hubs. The $\mathrm{CH}$ additionally goes about as one of the PKG serving hubs and assumes the job of key combiner. Private Key offers created by k PKG serving hubs are consolidated by $\mathrm{CH}$ to get entire key[5][6]. Beginning open key of $\mathrm{CH}$ is gotten by applying one way hash work on its id. $\mathrm{CH}$ open key fluctuates dependent on its trust level. New open key of $\mathrm{CH}$ is figured dependent on old pubic key and its new trust esteem. The private key of $\mathrm{CH}$ is at first appointed by system head.

Later private key offers are processed by PKG hubs. The general population key of $\mathrm{CH}$ is dispersed to all bunch individuals in the comparing group. The portable specialist is a program section that gathers data about $\mathrm{k}$ trustable hubs in a group and data about hubs whose declaration is revoked [8]. The framework utilize two dimension of recurrence for correspondence: low dimension recurrence is utilized to set up correspondence between bunch individuals and abnormal state recurrence is utilized to set up correspondence between bunch heads.

The zone based key administration conspire is a cross breed key administration blueprint for MANETs, in light of crafted by [2][3] and the Zone Routing Protocol (ZRP)[4], where a zone is characterized for every hub and incorporates the hubs whose remove (e.g., in jumps) is at most some predefined number. This separation is eluded to here as the zone range, rzone. Every hub utilizes symmetric key administration inside its zone and unbalanced key administration utilized for between zone securities, without relying upon bunching. For effortlessness and consistency, the documentation descried in [2]will be utilized in this work, except if generally determined

In Group Key Management Schemes, assemble key is an extraordinary key that is doled out to a gathering of hubs. So as to build up a gathering key, the gathering needs to make and disseminate the way to all individuals. 


\section{ASSAULTS ON MANET}

There are different sorts of assaults on specially appointed system:

\section{A. LOCATION DISCLOSURE}

Area exposure is an assault that objectives the protection necessities of a specially appointed system. By utilizing movement examination systems, more straightforward testing and observing methodologies, an assailant can identify the area of hub

\section{B. DARK HOLE}

In a dark gap a vindictive hub infuses false course answers to course asks for, reporting it as having the briefest way to a goal. These phony answers can be manufactured to redirect arrange activity through the vindictive hub for essentially to pull in all the movement towards it keeping in mind the end goal to play out a dissent of administration assault by disposing of the got bundles.

\section{REPLAY}

A replay assault is one of the assaults that debase extremely the execution of MANET. an answer assailant does this assault by block attempt and retransmission of substantial marked messages.

\section{WORMHOLE}

In a wormhole assault, an assailant gets bundles at one point in the system, "burrows" them to another point in the system, and after that replays them into the system starting there

\section{E. SHAKEDOWN}

These assaults are pertinent against steering conventions that utilization instrument for the recognizable proof of noxious hubs and engender messages and endeavor to confine honest to goodness hub from the system. The non-renouncement security criteria can end up being conveniently.

\section{F. DENIAL OF SERVICE}

Refusal of administration assaults go for the total disturbance of the directing capacity and along these lines the whole activity of the specially appointed system. In a directing tangle flood assault the vindictive hub surges the system with counterfeit course creation parcels to devour the assets of taking an interest hubs and upset the foundation of lawful course.

\section{G. DIRECTING}

Table harming Routing convention keeps up table that hold data with respect to course of the system. In this kind of assault, the vindictive hub create and send manufactured flagging movement or adjust honest to goodness message from alternate hubs ,with a specific end goal to include false sections in the tables of the taking an interest hubs.

\section{H. MASQUERADING}

Amid the neighbor securing process, an outside interloper could disguise a nonexistence or existing IS by assaulting itself to correspondence interface and unlawfully participating in the steering conventions area by including verification framework. The danger of disguising is nearly the same as that of a bargained IS.J.

\section{IMPERSONATION}

Pantomime assaults are a serious danger to the assailant can catch a few hubs in the system and influence them to look like cordial hubs. Subsequently, the bargained hubs can join the system as the ordinary hubs and start to lead the vindictive practices, for example, spread phony steering data and increase improper need to get to some classified data.

\section{J. LISTENING STEALTHILY}

It is another sort of assault that generally occurs in the versatile impromptu networks. Eaves droping intends to get some classified data that ought to be kept mystery amid the 
correspondence. The classified data may incorporate general society key, private key, area and passwords of the hubs. Since such information are exceptionally private to the hubs, they ought to be kept mystery with the goal that unapproved can't get to this.

\section{PROPOSED MODEL}

Following focuses are considered as pre-presumptions for outlining secure information transmission in bunch based WSN:

1. The hubs in the system framework are versatile with accessible locally available memory, figuring capacity, correspondence data transfer capacity, and accessible battery control.

2. Battery might be inexhaustible or not sustainable.

3. The sensor hubs are not solid and they should be confirmed.

4. The aggressors may listen in the radio transmissions. The aggressors can likewise send some indistinguishable hubs to misdirect genuine hubs.

For proposed calculation, 3 distinct kinds of keys are created i.e. Introductory mystery key (ISK), sensor-group entryway key (SCGK) and door base key (GBK) in proposed display.

Each sensor hub has an Initial Key (at first stacked into memory of every sensor hub) alongside their ID. Every sensor hub utilizes the ISK and ID at first to verify itself with base station.

SCGK key is utilized for the correspondence inside a group among bunch head and sensor hubs. SCGK is produced by base station alongside bunch ID and send to all group heads. This key is then disseminated among all hubs utilizing ISK.

GBK is utilized to convey between door hub and base station.

VENTURES OF PROPOSED CALCULATION:

1. The confirmation stage comprises of following advances:

Every hub send validation demand to base station. The ask for message contains ID encoded with ISK

Base station at that point decode ID utilizing ISK of hub

In the event that ID coordinated at that point confirmed and base station doles out SBK to every hub.

2. After organization of the sensor hubs in the field, the base station isolates the sensor field into a few bunches.

3. In each bunch relegate one Cluster Head Nodes (CH). All Sensor Nodes in a bunch speak with the Cluster Head Node lastly, the Gateway Nodes are in charge of transmitting information to the base station (as portrayed in underneath figure).

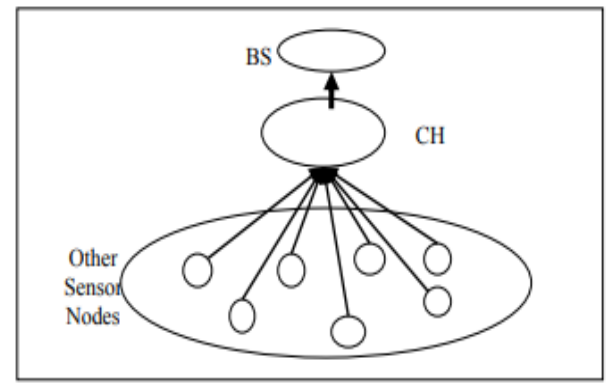

Figure 2. Proposed Model 
On the off chance that any Sensor Nodes sense information from nature then it advances the information to its separate Cluster Head hub. Advance the Cluster Head hub advances the information to the base station.

\section{CONCLUSION}

In this paper, a near investigation of remote sensor organize (WSN) directing conventions. The conventions are partitioned into two classifications i.e., bunch based and non-group based. It is watched that among all steering conventions group based conventions are various leveled, dependable, and vitality proficient directing convention that beats others. Thus, it can gathered that group based conventions are more mainstream among the examination network of WSN. One of the key issues in WSN is security issue in which verification is a principal issue for a dependable system benefit. The hubs must have the capacity to recognize dependable from deceitful hubs in the neighbor revelation process, and they should have the capacity to check both steering message beginning and respectability. Any cryptographic validation conspire requires appropriate key administration. That is, common verification of the included gatherings is required amid the key setup. This paper incorporates a thorough review of key administration techniques proposed for Adhoc systems. The appropriateness of personality based open key plans for assurance of Adhoc steering data is likewise talked about.

\section{References}

[1] Kenney, M. \& Zysman, J. “The rise of the platform economy”. Issues in Science and Technology, 32(3), pp.6169. (2016)

[2] G.Singla, M. S. Sathisha, A. Ranjan, S. D., and P. Kumara, "Implementation of protected routing to defend byzantine attacks for MANET’s," International Journal of Advanced Research in Computer Science, vol. 3, pp. 109, (2012).

[3] G.Singla and P. Kaliyar, "A Secure Routing Protocol for MANETs Against Byzantine Attacks," Computer Networks \& Communications (NetCom), Lecture Notes in Electrical Engineering, vol. 131, pp. 571-578, (2013). DOI: 10.1007/978-1-4614-6154-8_56

[4] X.Lv and H. Li, "Secure group communication with both confidentiality and non-repudiation for mobile adhoc networks," Information Security, IET, Vol. 7, (2013). DOI: 10.1049/iet-ifs.2010.0314

[5] S.a.A.k.G, H.o.d.R.m, and S. sharma, "A Comprehensive Review of Security Issues in Manets," International Journal of Computer Applications Vol. 69 (2013).DOI: 10.5120/12097-8277

[6] Balasubramanian A., Misha, S. and Sridhar, R.(2004). "A Hybrid approach to key management for enhanced security in ad hoc networks”. Technical report, University at Buffalo,NY, USA.

[7] Balasubramanian A., Misha, S. and Sridhar, R.(2005), “Analysis of a hybrid key management solution for ad hoc networks”. IEEE WCNC’05. Vol. 4, pp.2082-2087.DOI:10.1109/WCNC.2005.1424839

[8] Marjan Radi, Behnam Dezfouli, Kamalrulnizam Abu Bakar and Malrey Lee,” Multipath Routing in Wireless Sensor Networks: Survey and Research Challenges”, Sensors (2012), 12, pp.650-685. 